\title{
Tours 2030 : une vie pareille autrement... Étude sociologique sur les conditions d'acceptation d'un scénario « Ville post-carbone »
}

\author{
Estelle Durand ${ }^{a}$
}

CETU -ETIcS/Université François-Rabelais, Tours, France

\begin{abstract}
Résumé. La recherche "Conditions d'acceptation d'un scénario Vivre Post Carbone » interroge, sous l'angle sociologique, la réception, par les habitants des territoires concernés, du scénario « Vivre Post Carbone 2030 » pour le périmètre du SCoT (Schéma de Cohérence territoriale) de l'agglomération tourangelle (37). Une trentaine d'entretiens qualitatifs ont été réalisés auprès de ménages tourangeaux d'âges, de niveaux de revenus et de lieux de résidence différents. La question des impacts et des coûts sociaux du scénario, plus globalement de son acceptabilité par les habitants, constitue la trame des résultats. Le scénario est, en effet, diversement reçu par les interviewés en fonction de leurs contraintes objectives et de la perception de leurs propres marges de manœuvre. Dans tous les cas, les éléments du scénario apparaissent comme d'autant plus crédibles, acceptables et « désirables » qu'ils convoquent des échelles de temps et d'espace appropriables par les individus. Loin d'adopter une position d'objection ou d'hostilité de principe à tout changement, les ménages rappellent que l'enjeu principal pour eux reste le maintien et/ou l'amélioration de leurs cadres et modes de vie.
\end{abstract}

\begin{abstract}
The survey "Acceptance conditions of a Post Carbon Living scenario" adopts a sociological perspective, in order to question the reception of the scenario "Vivre Post-Carbone 2030" for the SCOT perimeter (territorial coherence scheme) of Tours agglomeration by the inhabitants of the territories concerned. About thirty qualitative interviews were conducted with households from Touraine of different ages, income levels and places of residence. The matter of social impacts and social costs of the scenario - more generally of its acceptability by the people - is the background of the results. Indeed, the scenario is differently received by interviewees, according to their objective constraints and the perception of their own room for maneuvre. In all cases, the scenario elements appear more credible, acceptable and "desirable" when they convene time and space scales which can be appropriated by people. Rather than adopting an objection or hostility position to change, households point out that their main challenge remains to maintain and/or improve their living environment and lifestyle.
\end{abstract}

\footnotetext{
${ }^{\mathrm{a}} \mathrm{e}$-mail : estelledur@gmail.com
}

This is an Open Access article distributed under the terms of the Creative Commons Attribution License 4.0, which permits unrestricted use, distribution, and reproduction in any medium, provided the original work is properly cited. 


\section{SHS Web of Conferences}

\section{En préambule...}

La recherche «Axes de progrès pour un SCoT Facteur 4 : Quels leviers locaux pour une agglomération post-carbone ? » réalisée par l'Agence Tourangelle d'Urbanisme (ATU) et Beauvais Consultants a porté sur les conditions d'une transformation en profondeur des structures et du fonctionnement des villes et d'une transition vers des villes post-carbone. Villes dont l'impact en termes d'émissions de gaz à effet de serre et de consommation de pétrole ou de gaz est considérable. À travers la diversité des fonctions urbaines (habitat, commerces, services, loisirs, emploi, etc.), la ville des proximités et le modèle de la ville dense-compacte sont au cœur des réflexions sur l'autonomie énergétique du territoire. L'objectif de cette recherche est de définir le chemin à parcourir pour atteindre le Facteur 4 en 2050, en utilisant comme principal levier l'aménagement de l'espace et en bâtissant un scénario situé à un horizon intermédiaire, celui de 2020-2030.

Cette première étude a permis la production d'un scénario Vivre Post Carbone 2030 pour le périmètre du SCOT (Schéma de Cohérence territoriale) de l'agglomération tourangelle ${ }^{1}$ [1]. Construit conjointement par l'ATU (Agence Tourangelle d'Urbanisme) et Beauvais Consultant, il explore quatre pistes « pour un développement urbain guidé par le principe de proximité bien vécue ».

- Un recyclage urbain avec une ville qui se reconstruit sur elle-même en exploitant des espaces devenus obsolètes (zones commerciales, zones d'activités) et en densifiant les espaces habités (démarche BIMBY, Built in my Backyard) tout en respectant la mixité fonctionnelle (1/3 habitat, $1 / 3$ activités, $1 / 3$ espaces naturels).

- Des mobilités repensées, des distances raccourcies grâce à la ville des proximités.

- Des surfaces agricoles et naturelles préservées et valorisées à partir du principe « zéro hectare » en extension urbaine pour les nouveaux aménagements, une agriculture périurbaine de proximité constituant un contrepoids à la ville dense.

- Une démarche globale de maîtrise des énergies pour le résidentiel comme pour les activités tertiaires et industrielles, le recours aux énergies renouvelables.

Parallèlement, la recherche sociologique « Conditions d'acceptation d'un scénario Vivre Post Carbone » vise à confronter les éléments du scénario conçu par l'Agence Tourangelle d'Urbanisme (ATU) et Beauvais Consultants dans le cadre de l'appel à projet Ademe «Ville post-carbone » aux pratiques et aux représentations des ménages. Il s'agit d'accéder à la perception des impacts potentiels du scénario ainsi qu'aux marges de manœuvre et aux leviers d'actions du point de vue des habitants du SCoT (Schéma de Cohérence Territoriale), territoire concerné par le scénario.

Cette recherche a constitué une sorte de «prolongement sociologique » permettant d'accéder à la manière dont les ménages de l'agglomération tourangelle se projettent et s'approprient les différents éléments du scénario Vivre Post Carbone 2030. La question des impacts et des coûts sociaux du scénario, plus globalement de sa recevabilité, sa compréhension et son acceptabilité par les habitants des territoires concernés, constitue la trame du questionnement.

L'objectif est bien de confronter les pistes explorées à travers le scénario aux pratiques et représentations des ménages et de mettre en évidence la manière dont ils se projettent et s'approprient un scénario qui les invite à reconsidérer leurs modes de vie et leurs modes de consommation actuels. Cette recherche appréhende, dans leur imbrication, la thématique de la mobilité (organisation des déplacements - actuelle et envisageable - au regard du scénario) et celle de l'habitat, du parcours et des choix résidentiels (dimensions réformables ou non au regard du scénario). L'évocation de ces deux thématiques permet également d'aborder les activités, loisirs et achats et d'évaluer ce que le scénario pourrait avoir comme conséquence sur leur gestion et leur évolution.

\footnotetext{
${ }^{1}$ Axes de progrès vers un schéma de cohérence territoriale Facteurs 4. Quels leviers locaux pour une agglomération postcarbone ?, Rapport Final, ATU, Beauvais Consultants, Programme Villes post-carbone, ADEME, janvier 2011.
} 
Il s'agit d'identifier les changements socialement acceptables, de repérer et de donner du sens aux obstacles et aux freins, repérés ou exprimés. Invitant les interviewés à se livrer à un exercice de projection, la recherche vise à identifier les leviers sur lesquels s'appuyer pour dépasser ces obstacles et ces freins, au regard du scenario construit.

De même, saisir finement les leviers d'actions possibles renseigne sur l'appropriation du scénario, sur la projection possible. Comment la modification des comportements peut-elle se traduire concrètement? Les changements envisagés se situent-ils au niveau résidentiel, modal, des activités ou professionnel ? Dans l'imbrication de ces diverses dimensions ? À quelles temporalités : court, moyen ou long terme?

L'impact carbone ne se réduit pas uniquement à la dimension «mobilité » mais intègre également la dimension «bâtiment». Pour comprendre les réformes possibles, les enjeux énergétiques doivent être appréhendés dans la transversalité et l'imbrication de ces dimensions. Nous avons tenté de comprendre comment les ménages raisonnent et si, dans leurs perceptions, projections et arbitrages, ils distinguent les impacts carbone «bâtiment » et les impacts carbone «mobilité ». Autrement dit, cloisonnent-ils et prennent-ils des mesures dans un domaine ou un autre ou estiment-ils devoir agir dans les deux ? Redoutent-ils d'agir sur l'un, par conscience ou crainte que l'autre soit inévitablement impacté ? Il s'agit notamment d'évaluer les risques d'effets rebonds directement liés à des changements, plus globalement à la confrontation des différentes rationalités et «paradoxes » de l'efficacité énergétique ${ }^{2}$ [2].

Pour saisir ces interactions, nous nous sommes attachés à repérer les « micro-ajustements ». Souvent analysés comme des réponses partielles et de faible portée par l'observateur extérieur, ils correspondent pourtant, du point de vue des ménages, à de profonds changements, voire à des ruptures. Ils interrogent ce qui relève des initiatives possibles et envisageables, tout autant à l'échelle individuelle et de la sphère privée qu'à l'échelle sociale et collective. Ces niveaux d'échelle permettent de saisir des formes d'interaction, d'implication et de mutualisation sur lesquelles les individus et foyers interrogés se projettent diversement et pour lesquelles ils disposent de marges de manœuvre inégales.

\section{La méthodologie}

Les recueils de données ont été effectués auprès de trente ménages résidant dans les quatre grandes zones du périmètre du SCoT. Ces quatre zones renvoient à autant de situations résidentielles, de modes de vie et d'organisations modales :

- Des ménages du cœur métropolitain de la ville centre (mésopotamie, entre Loire et Cher) ;

- Des ménages du reste du cœur métropolitain et du Noyau Urbain (Centre-Reste noyau urbain) ;

- Des ménages de périphérie (couronne proche);

- Des ménages de périphérie (couronne lointaine) $)^{3}$.

\footnotetext{
2 A. Dujin, B. Maresca, La température du logement ne dépend pas de la sensibilité écologique, CREDOC revue « consommation et modes de vie », $\mathrm{n}^{\circ} 227$ (mars). "La facilité plus grande à maintenir à un niveau élevé la température des pièces du logement, du fait d'une isolation et d'une ventilation plus performantes, pousse les usagers à accroître leur niveau de confort. (...) La rationalité économique des entreprises et des gestionnaires d'immeubles, (. ..) atteint plus difficilement son but pour les ménages car elle ne correspond pas à leur logique d'arbitrage, qui ne va pas spontanément vers la recherche d'économies financières. » (2008).

${ }^{3}$ Lors du dépôt de notre proposition, nous avions envisagé d'interviewer des ménages de banlieue parisienne. Nous faisions l'hypothèse que les changements inscrits dans un scénario «Ville post-carbone » pouvaient renforcer l'attractivité de la Touraine (plutôt que la périphérie de Paris). Il nous est finalement apparu plus pertinent de concentrer les recueils sur des foyers résidant tous en Touraine (périmètre SCOT) afin de pouvoir mettre en perspective les éléments contextuels et territoriaux. Le « risque » était notamment que les foyers parisiens convoquent des motivations à déménager que nous ne retrouverions pas chez les autres foyers.
} 


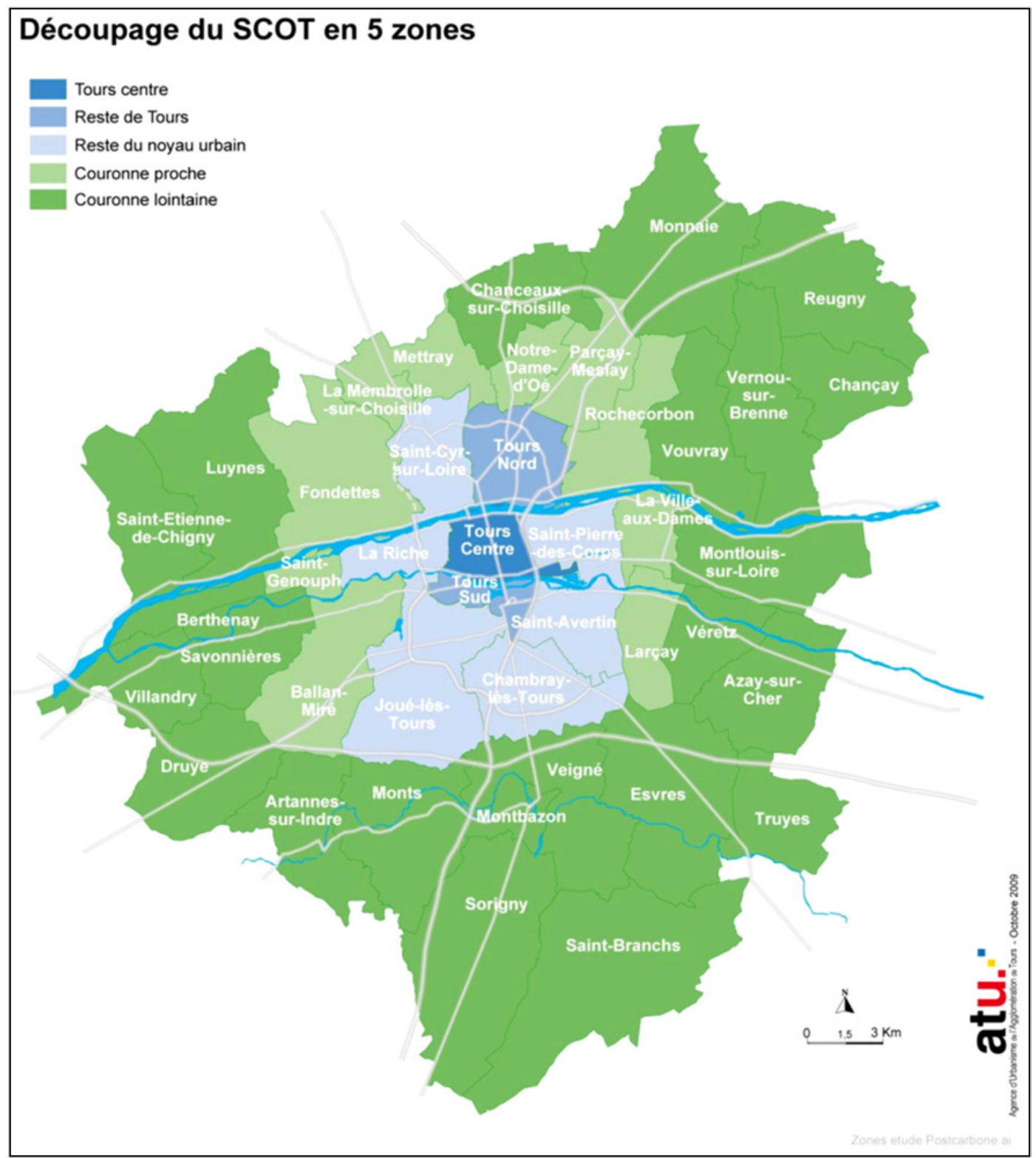

À chaque fois que ceci a été possible, la collecte des données a été effectuée auprès du ménage. Plutôt que de recueillir le point de vue d'un individu, nous avons raisonné à l'échelle du foyer afin d'accéder à l'impact potentiel du scénario sur ses différents membres. Les transformations inscrites dans le scénario «Ville post-carbone » agissent sur les organisations familiales en tant qu'entité, tout autant que sur les individus qui la composent. Les modalités d'implantation territoriale et de gestion des déplacements se construisent sur des représentations et occasionnent des négociations pour lesquelles il est pertinent de recueillir la perception du couple et, quand cela est possible, celle des autres membres du foyer.

Bien que les thématiques abordées aient été communes pour tous les interviewés, la conduite de l'entretien a tenu compte de la diversité des lieux de résidence. Les ménages qui résident dans le noyau urbain peuvent-ils envisager de céder à la faible densité et décider, même temporairement, de s'éloigner 
de la ville dense et d'opter pour un type et un lieu d'habitat offrant un meilleur cadre de vie ? Pour les ménages des couronnes proche et éloignée, la problématique se pose autrement. Une partie de ces ménages argumente le choix résidentiel par la volonté de disposer d'un cadre et d'un espace de vie satisfaisants. Et même si travailler dans la ville centre peut être pesant (impact de l'éloignement, contraintes de temps et de coût, niveau de motorisation...), résider en périphérie leur permet de maintenir une rupture entre espaces/temporalités de travail et espaces/temporalités hors-travail. Pour d'autres ménages, l'implantation en périphérie relève plus d'une contrainte, notamment de coût du foncier. Pour ces ménages de périphérie, l'analyse a largement abordé les conditions d'attractivité d'une ville post-carbone.

L'échantillon construit n'est pas un échantillon représentatif au sens statistique du terme mais un échantillon raisonné à partir de variables pertinentes au regard des objectifs de la recherche.

Les ménages ont été sélectionnés selon un ensemble de caractéristiques cohérentes au regard des objectifs de l'étude :

- Composition de la cellule familiale : nombre de personnes, présence (ou non) d'enfants. De précédentes recherches ont montré que la présence d'enfants au sein du foyer intervenait, parfois fortement, dans les choix résidentiels et dans l'organisation, notamment modale, des activités ${ }^{4}[3,4]$;

- Âge/cycle de vie des personnes interviewées : activité, âge des enfants, situation des personnes au regard de l'emploi/études... La projection dans des organisations plus ou moins synonymes de ruptures, tout du moins de changements importants, est très liée au contexte de production des discours. Le «moment» du cycle de vie renseigne sur l'opportunité de changement que peut constituer le scénario en construction. Selon le moment de leur vie professionnelle, de leur parcours résidentiel (en phase de construction, ascendante ou incertaine), les ménages se sont saisis des réflexions inscrites dans le cadre de « la ville post-carbone » pour élaborer, concrétiser, voire repenser un projet professionnel et/ou résidentiel. À l'inverse, d'autres ménages ont pointé l'incompatibilité entre ces réflexions et leur projet de vie ;

- Profession et Catégorie Sociale (PCS) du ménage. Les interviewés disposent, dans des conditions inégales, de marges de manœuvre pour envisager une transformation de leurs organisation et mode de vie. La prise en compte de cette diversité de ressources et de capitaux est indispensable pour appréhender le plus largement possible le spectre des réactions au scénario proposé.

Les entretiens ont été consacrés à l'organisation des ménages, notamment sur le plan modal (indication du niveau de motorisation, gestion des mobilités), aux déplacements effectués (à l'échelle d'une journée/semaine). Les choix résidentiels (critères en termes de cadre et de qualité de vie, présence d'un réseau familial/amical, absence de choix - contrainte de coût du foncier) ont également été interrogés. Ces informations ont permis d'appréhender le niveau d'attachement au lieu de résidence, les aspects qui ont prévalu à l'implantation. Elles ont aussi permis d'identifier les marges de manœuvre existantes pour envisager une alternative, aussi bien sur le plan organisationnel (modal) que résidentiel.

Ainsi, pour chacun des ménages, l'objectif était de pouvoir :

- apporter des éléments de réflexion à la problématique énergétique «carbone \& ménages ». Il s'agissait d'analyser les formes de perméabilité entre les réflexions et les actions portant sur le carbone «bâtiment » et celles portant sur le carbone « mobilité »;

- mesurer les évolutions susceptibles d'être acceptées au sein de la sphère privée, et accéder aux négociations qui ont cours entre les membres du foyer. De même, les temporalités pour lesquelles

\footnotetext{
${ }^{4}$ Signal Prix et arbitrage de moyen et long termes. Comportements des familles face à la hausse du prix du carburant, en collaboration avec Beauvais Consultants, PREDIT, (2009). Habiter à Tours et travailler à Paris. Étude sur les migrants professionnels, en collaboration avec Beauvais Consultants, PREDIT/DRAST (2007).
} 
des modifications importantes semblaient envisageables, par exemple un déménagement, ont fait l'objet d'échanges. Ce qui apparaît impensable aujourd'hui (enfants trop petits, situation professionnelle trop hypothétique, revenus trop faibles...) peut le devenir à une échéance plus ou moins proche (recomposition de la sphère familiale, évolution sur le plan professionnel et des revenus...);

- identifier les attentes des ménages envers les politiques publiques pour des modes de vie décarbonés sur le territoire. À travers l'évocation de leur organisation, des avantages et contraintes liés à leur localisation résidentielle, les ménages ont abordé l'action des acteurs politiques. Ils ont été invités à commenter les réflexions et le scénario en cours, les limites entrevues mais aussi les transformations positives.

$\mathrm{Au}$ total, une trentaine d'entretiens ont été réalisés auprès de ménages résidant dans l'agglomération tourangelle en respectant une répartition par niveau de revenus, âge et composition du foyer et entre habitants de l'hyper-centre ville, du reste de la ville de Tours, de la couronne proche et de la couronne lointaine.

\section{Les principaux enseignements}

\section{Le scénario Vivre Post Carbone 2030 est lu à l'aune des modes de vie actuels et anticipés}

Les ménages ont éprouvé des difficultés à appréhender le scénario dans sa globalité et sans doute également dans sa complexité. Pour faciliter la projection dans le scénario, ce dernier a été présenté aux interviewés sous forme de thématiques dont nous espérions qu'elles seraient reconstruites pour envisager le changement sous un angle global. Il apparait, au final, qu'il n'est pas si simple d'appréhender le changement comme un ensemble cohérent. Les réactions recueillies montrent que si certaines thématiques sont naturellement associées, par exemple le choix résidentiel et les mobilités, d'autres apparaissent comme plus atomisées. Par ailleurs, les thématiques ont été très diversement appropriées et ont donné lieu à plus ou moins de discours. La capacité à se projeter dans un « autrement » et la perception de ses propres marges de manœuvre ainsi que le caractère polémique de certaines propositions du scénario ont conféré un poids plus ou moins important à chacun des leviers du changement.

Saisi au travers de thématiques, il n'apparait pas comme un scénario de rupture mais plutôt comme un avenir prévisible auquel il faut s'adapter. Dès lors, les éléments du scénario sont appréhendés au travers du prisme du mode de vie actuel, résultat de multiples contraintes, arbitrages et auquel les ménages sont attachés. Cette lecture est fortement induite par l'ancrage territorial. La perception des marges de manœuvre est, en effet, étroitement liée aux effets de trajectoire, aux choix résidentiels et aux ressources en termes de mobilité. La position dans l'espace social, ici la capacité à bénéficier des changements induits par le scénario et/ou s'en affranchir si nécessaire, joue un rôle important dans la manière de se positionner par rapport au scénario. Cette position est également structurée par les évolutions prévisibles (enfants qui grandissent, vieillissement, mobilité résidentielle prévue ou souhaitée...) et une certaine lecture du passé permettant d'interpréter les injonctions au changement, plus particulièrement la sobriété énergétique ou encore le réinvestissement des circuits agricoles courts.

Deux facteurs principaux structurent les discours recueillis et orientent la perception du scénario «Vivre Post Carbone».

Le premier facteur est en lien direct avec la position que les interviewés occupent dans l'espace social. La lecture du scénario est alors fortement dépendante des marges de manœuvre existantes ou encore de la capacité à agir. Selon qu'ils s'estiment en capacité de s'approprier les éléments du scénario, de mettre en place les changements qu'il sous-tend, ou au contraire dans l'impossibilité de s'en affranchir, les interviewés livrent une analyse construite sur le registre de la contrainte ou sur celui 
des opportunités induites. Le clivage se fait alors entre une lecture «positive » d'un scénario qui peut offrir des bénéfices sur le plan de l'organisation et des modes de vie et une lecture plus nuancée dans laquelle les discours se focalisent sur ses répercussions « négatives » pour l'ensemble de la cellule familiale ou pour certains de ses membres.

Le deuxième facteur est en lien direct avec la situation géographique et résidentielle des interviewés. Les arguments avancés se fondent sur l'incidence du scénario sur la gestion, organisationnelle et temporelle, des déplacements. Selon que les changements retravaillent les notions de proximité ou d'éloignement d'avec les lieux d'activités et impactent - positivement ou négativement - les modes de vie, les interviewés livrent un discours d'adhésion ou d'opposition au scénario. Les notions de confort et de qualité de vie, les « choix » en termes de localisation résidentielle deviennent des éléments de débat, voire de divergence, entre les différents profils d'interviewés.

Position dans l'espace social, situation géographique..., les ménages interviewés s'interrogent sur l'impact du scénario «Vivre Post Carbone » sur leurs modes de vie. Que de l'extérieur l'organisation mise en place apparaisse satisfaisante ou non, cohérente ou pas, les ménages s'y montrent fortement attachés. Cet attachement ne doit pas être interprété comme une résistance au changement ou une incapacité à réformer les comportements. Cette organisation est le résultat de multiples arbitrages, la plupart du temps complexes, parfois de concessions - socialement, symboliquement, économiquement coûteuses. Bien que déjà en place, elle demeure souvent décrite comme un équilibre fragile et instable. Maintenir ces modes de vie constitue le point de départ et l'ossature de la réflexion. Les arguments mobilisés se construisent à partir de ce « fil conducteur » dont les interviewés ne se départissent pas ou difficilement.

\section{La mobilité, un thème particulièrement investi par les interviewés}

La mobilité est sans doute le thème le plus développé par les interviewés parce que le plus immédiatement impactant sur les organisations. Elle engage des réorganisations et des incertitudes, projette dans des arbitrages, voire des renoncements de choix de vie. C'est aussi le plus complexe en termes de projection. Cette complexité s'explique non pas par un attachement culturel à la voiture par exemple, mais parce que cette thématique est déjà explorée depuis longtemps.

La maturité sur la question des mobilités s'explique par l'obligation pour les ménages de trouver une organisation satisfaisante, du point de vue de la qualité de vie et des contraintes à gérer. Qu'il s'agisse d'un changement de transport ou encore d'une organisation des déplacements qui a pu être repensée, la plupart des familles rencontrées ont testé un « autrement». Cela est en particulier vrai de celles qui résident à la campagne ou en grande périphérie, conscientes de leur dépendance à la voiture individuelle et désireuses d'en sortir, au moins de la réduire. Pour celles qui ont testé les déplacements habituels à partir de l'offre en transports collectifs, l'arbitrage s'est fait autour de la notion de temps. L'organisation trouvée devait permettre de gérer dans des conditions quasi-identiques les besoins de déplacements. Se révélant beaucoup plus chronophage, cette nouvelle organisation a souvent été abandonnée.
"J'ai fait aussi, voiture-bus (...) On part tôt le matin déjà et on met du temps à rentrer chez soi le soir. Jusqu'à temps que j'arrête de travailler sur Tours, avec mon mari, on comptait qu'on était parti 11 heures par jour. » (Nadine, Couronne lointaine)
"C'était un cauchemar par rapport aux horaires, par rapport au peu de bus, et j'ai même dî faire un changement de bus et ça m'a bien agacé. C'était beaucoup plus long qu'en voiture, entre le temps d'attente à l'arrêt de bus, le temps qu'il fasse toutes les petites rues dans Saint Avertin, que je change que ceci que cela, j'ai mis beaucoup plus temps. »(Alex, Couronne lointaine)

La question de l'habitat fortement lié à la mobilité renvoie spécifiquement au mode de vie et suscite immédiatement des appréhensions. Les « choix » résidentiels sont rationalisés, construits sur un fort ancrage local. Sont à l'œuvre d'autres rationalités que la rationalité économique. Le déménagement est 
rarement envisagé, et même s'il l'est, les interviewés se situent à des échéances lointaines et fortement dépendantes de décisions fragiles et incertaines (orientation scolaire ou professionnelle d'un enfant, activité professionnelle, problématique de santé et d'autonomie). Concernant le bâti, c'est avant tout la notion de confort qui est invoquée. Les économies d'énergie sont souvent appréhendées après les gains en confort. La relation entre habitat et énergie n'est d'ailleurs pas systématique. Viennent ensuite les modes de consommation mais dans ce domaine, les interviewés se sont surtout exprimés sur leur impuissance à agir en dehors de la réappropriation de certaines filières courtes et de la possibilité, sous condition, de mutualiser quelques équipements. L'enjeu transversal restant le maintien du mode de vie, seuls sont acceptés les changements qui ne le remettent pas fondamentalement en cause.

\section{La maîtrise des échelles du changement, une condition d'adhésion au scénario}

Le scénario propose des objectifs à atteindre, identifie les leviers prioritaires du point de vue des collectivités locales, engage une réflexion sur les changements attendus mais donne peu de clés de lecture sur le rôle des habitants, pourtant central. Sans être pessimistes, les interviewés interrogent le scénario sous deux angles complémentaires : Les échelles pertinentes de l'action, les territoires concernés et les échéances temporelles.

Les éléments du scénario apparaissent comme d'autant plus crédibles, acceptables et désirables qu'ils convoquent des échelles de temps et d'espace qui font sens et qui apparaissent appropriables par les individus. L'appropriation des axes du scénario et ses projections associées s'inscrivent toutefois sur des temporalités distinctes.

Les interviewés convoquent deux niveaux de lecture. Le premier niveau de lecture concerne les changements qui peuvent s'inscrire rapidement dans les modes de vie actuels dans lesquels les interviewés se projettent avec une plus grande facilité, intégrant immédiatement les possibles conséquences sur leurs modes de vie. Le second niveau de lecture renvoie aux éléments du scénario qui, sans faire l'objet d'une opposition formelle, vont nécessiter un temps d'appropriation plus long dans la mesure où ils engagent une réflexion collective et des formes de régulation sociale qui échappent à l'individu. C'est souvent cette difficulté à se projeter, et à évaluer le temps nécessaire à cette projection, qui explique les positions de craintes, dans certains cas les objections. Plus qu'une posture de résistance de principe, c'est l'exercice projectif qui perturbe.

- Le degré de responsabilité de chacun des acteurs, les marges de manœuvre individuelles et collectives mobilisables pour engager des changements significatifs alors même que certains enjeux échappent partiellement ou totalement aux individus (enjeux économiques, stratégies industrielles, investissement d'infrastructure...).

De fait, ils interrogent le rapport individu/société et ce que cela engage concrètement du point de vue des organisations collectives, des conditions de contrôle et de régulation sociale.

Les éléments du scénario apparaissent comme d'autant plus crédibles, acceptables et désirables qu'ils convoquent des échelles de temps et d'espace appropriables par les individus. Ainsi, c'est l'échelle locale, celle de la proximité qui est plébiscitée (le « quartier, le « village »). La relation au collectif se construit alors dans une logique élective. Les rapports sociaux sont maîtrisés, les organisations collectives se déploient sur la base de la réciprocité. La régulation sociale est garantie par l'intériorisation de normes et de valeurs partagées.

À travers la lecture du scénario et des exemples qu'ils convoquent, les interviewés interrogent, audelà même des conditions d'adhésion, la dualité entre le proche et le lointain. La notion de proximité et de « proche » renvoie à ce qui est faisable et maîtrisable. Au sein des représentations, elle correspond aux choix individuels. Alors que le « lointain » est perçu à la fois comme contraignant, difficilement maîtrisable et d'une certaine manière difficilement réalisable. Le plus souvent, il est associé aux 
impératifs du collectif, et plus globalement à des intérêts potentiellement concurrents à ceux des ménages.

Ainsi, l'articulation entre les marges de manœuvre et la perception des contraintes actuelles et anticipées renvoie à la complexité des rapports entre l'individuel et le collectif, entre l'échelle de la communauté et celle de la société.

La communauté protège, préserve les individus. Les membres d'une communauté font partie d'un tout jusqu'à se confondre avec ce tout (la famille, le village, la corporation). La communauté crée du sens, et ce sens est la base du droit qui régit la vie commune des membres du groupe. La société crée un nouvel ordre et élabore un projet de vie commune. Elle rassemble des individus obéissant aux mêmes règles sans pour autant partager nécessairement un mode de vie commun. Même si les concepts de communauté et de société désignent deux façons différentes de vivre ensemble, elles restent complémentaires et s'articulent l'une à l'autre.

Ces deux niveaux d'appréhension de l'organisation des rapports humains et sociaux correspondent également à des niveaux différents d'action et d'adhésion aux éléments proposés par le scénario «Vivre Post Carbone».

À l'échelle de la communauté, c'est-à-dire du proche, de l'individu, de l'entourage, du quartier, du village, la contrainte sociale s'effectue à travers le regard de l'Autre. L'adhésion au changement fait directement écho à la responsabilité individuelle ou à celle des collectifs informels (l'entourage par exemple). Il s'agit alors d'un contrôle de type personnel, impliquant directement l'individu, l'incitant et l'accompagnant dans l'évolution possible de ses marges de manœuvre.

À l'échelle de la société, les règles sont plus formelles. L'adhésion inclue et mobilise le contrôle des pouvoirs publics. Il s'agit alors davantage soit de contraindre sous un mode culpabilisant et stigmatisant, soit d'accompagner en incitant. À ce niveau, les interviewés font référence à la régulation sociale comme levier de changement possible. Ce niveau d'adhésion est perçu comme le plus structurant mais également celui qui échappe le plus à l'arbitrage et à l'approbation des individus car plus abstrait. Comme le soulignent les verbatim suivants, les changements sont possibles si les alternatives sont pensées au préalable par les pouvoirs publics, les collectivités, et si la contrainte et les interdits sont clairement affichés. Voire, dans certaines situations précises, imposés d'un point de vue juridique.

\begin{abstract}
"On est allé à Bologne où les gens savent qu'effectivement, en dehors de certains horaires, ils ne peuvent pas circuler au cœur de ville. Ils laissent leur voiture sur un parking relais et après ils prennent le bus. Ça existe déjà et ça n'a pas pour autant tué l'activité du centre-ville. Il est évident que pour interdire la circulation effectivement dans le centre d'une ville, il faut développer parallèlement des alternatives. Ce qui n'existe pas aujourd'hui. » (Annie, Couronne proche)
\end{abstract}

«Pour moi, c'est uniquement par la contrainte... si on doit faire quelque chose, c'est par la contrainte. Les pistes cyclables à Paris, tout le monde était contre. Ça a été un tollé général et ceux qui utilisent les vélos trouvent cela génial et malgré tout, tout le monde dit quand tu vois la rue de Rivoli, c'est quand même vachement plus sympa maintenant. Avant, c'était irrespirable. Maintenant, c'est génial. (...) Si dans ta rue, tu as beaucoup plus d'espaces verts, que ça ne pue pas, qu'il n'y a pas de bruits, forcément, tu vas t'en rendre compte, mais pas tout de suite. Donc pour moi, ça passe par la contrainte. Une collectivité, c'est uniquement par la contrainte. »(Magalie, Mésopotamie)

Cette enquête confirme la difficulté des ménages à appréhender le changement à des échelles de temps, d'espaces et d'action qui engagent un collectif abstrait, et qui renvoient à des niveau de responsabilité et à des modalités d'action qui échappe à l'individu.

\title{
Le scénario « désirable » : une ville pareille autrement
}

L'aspiration à une meilleure qualité de vie constitue le moteur principal du changement vers une ville post-carbone. L'amélioration de la qualité de vie passe d'abord par la maîtrise du temps et de son 
contenu : les mobilités contraintes sont réinvesties d'un sens positif. Les trajets domicile-travail/école deviennent des temps libérés, les nuisances urbaines sonores et olfactives sont diminuées, les obligations telles que les achats alimentaires ou les démarches administratives sont réduites par la proximité et la possibilité d'en rationaliser la réalisation. Le périurbain devient un espace pertinent de développement, les centres urbains bénéficient d'un accès facilité par des modes de transport adaptés, les interactions ville/campagne sont apaisées.

On voit transparaître une attente forte autour du maintien et/ou de l'amélioration des modes de vie actuels. C'est en ce sens que les interviewés développent une lecture ambivalente des objectifs proposés par le scénario. L'injonction à la sobriété peut, par exemple, être perçue comme une opportunité de se réapproprier certains leviers d'action tout en inscrivant son mode de vie dans une démarche de responsabilité collective (sortir du caractère captif de la consommation d'énergie par exemple). Dans le même temps, elle peut apparaitre comme contraire au maintien d'un standard de confort qui caractérise le mode de vie auquel les ménages ne souhaitent pas renoncer.

L'injonction à la sobriété peut être favorablement accueillie dès lors qu'elle invite à retravailler les formes de coopération et d'échanges. Les interviewés entrevoient le scénario comme un levier à s'approprier, sans pour autant ressentir une remise en cause profonde de leurs modes de vie, plus encore de leur qualité de vie. Mais cette injonction suscite également des réactions, voire des formes de contestation qui relativisent l'adhésion au scénario.

L'ambivalence autour de la notion de sobriété vient de la difficulté à évaluer concrètement les gains et les pertes potentiels, en particulier à l'échelle individuelle, celle de la cellule privée. Les avantages sont assez rapidement perçus quand il s'agit d'impulser une approche collective des échanges, de la gestion en matière de déplacements ou de services. L'analyse des discours montre immédiatement que l'évaluation est plus nuancée, l'adhésion plus relative quand les éléments du scénario semblent atteindre les standards de vie et de confort.

De la même manière, la densification urbaine est immédiatement comprise comme la possibilité de retravailler les notions de proximité et d'accessibilité tout en renvoyant à l'idée de promiscuité et de réduction des espaces préservés (en particuliers jardins publics et privés).

Si le principe de la densification n'est pas exclu, la ville dense décrite doit concilier un besoin de loger les habitants, y compris les nouveaux venus, avec un désir d'habiter. Les périodes de fortes densifications sont alors convoquées comme autant d'expériences parfois malheureuses et qui n'ont pas été sans conséquences sur les cohabitations et les relations sociales.
«Aujourd'hui dans les habitats individuels et dispersés, on voit bien que les gens ont de plus en plus de soucis pour se chauffer. Revenir à des formes de densification serait donc normal mais il faut faire attention à quel type de densification on propose. Densifier ça ne veut pas dire construire comme dans les années 60 et construire en hauteur, en urgence parce qu'il fallait loger les gens en masse. Mais aujourd'hui il y a aussi un déficit de logement et on est tenté aussi de densifier mais il faut que cela reste raisonnable. »(Thierry, Mésopotamie).
« Des HLM construits sur la rue avec les fenêtres qui donnent sur la rue avec peu de trottoir... Ça rend la ville bizarre, ça fait un fortin. Même si j'ai bien conscience qu'il faut construire pour pouvoir loger les gens et surtout les jeunes, il ne faut pas malgré tout faire n'importe quoi en termes d'occupation de l'espace. Il ne faut pas que cela fasse kolkhoze. »(Christian, Mésopotamie)

La ville « désirable » est celle qui permet de concilier les dimensions fonctionnelles associées au territoire et aux politiques publiques et les attentes pratiques et symboliques des habitants en termes de mode de vie.

Lorsque l'on fait l'exercice de mettre en perspective les thématiques prioritaires du point de vue des concepteurs du scénario avec ce qui a effectivement retenu l'attention des ménages, on mesure bien toute la distance qui sépare la vision institutionnelle, nécessairement planificatrice, des aspirations des ménages. Le scénario propose des objectifs à atteindre, identifie les leviers prioritaires du point de vue 
des collectivités locales, engage une réflexion sur les changements attendus mais donne peu de clés de lecture sur le rôle des habitants, pourtant central.

\section{Des risques perçus : concurrence entre territoires et disqualification sociale}

Si pour les interviewés le scénario renvoient à des changements positifs, il induit aussi un certain nombre de risques sociaux. Ces risques sont appréhendés différemment en fonction des lieux de résidence (urbain/périurbain) et de la perception des marges de manœuvre que chacun peut mobiliser face aux changements induits par un scénario post-carbone.

La ville telle qu'elle est pensée risque d'amplifier les phénomènes de concurrence entre territoires et entre groupes sociaux en exacerbant les oppositions déjà bien réelles entre centre et périphérie. Plus qu'il ne le résout, le scénario renforce le sentiment de privatisation, de fermeture partielle de la ville aux habitants des périphéries. La « ville des proximités » semble alors ne s'adresser qu' aux urbains, les périurbains et les ruraux en étant écartés ; l'amélioration de l'offre de transport se révélant peu crédible du point de vue des ménages concernés.

En effet, à un premier niveau de lecture, le scénario risque d'amplifier les phénomènes de concurrence et d'opposition entre territoires, entre groupes sociaux et finalement entre individus. La ville identifiée et construite par le scénario correspond à une ville fermée et repliée sur son centreville. Dès lors, elle ne s'adresse plus à une collectivité territoriale et sociale harmonieuse mais à un groupe restreint. Cette représentation de la ville n'est plus celle de la ville des proximités, voire de la mixité sociale, mais celle des oppositions, des relégations, de la séparation entre le dedans et le dehors, entre l'admis et l'exclu/à exclure. La ville se renferme sur sa population « d'urbains faisant preuve d'urbanité » et exclut les habitants des périphéries, les périurbains et les ruraux. Ainsi, le territoire du SCoT ne correspond pas à un territoire apaisé car maîtrisé, contraint. Il semblerait, au contraire, que l'organisation sociale proposée par le scénario soit entendue comme facteur d'exacerbation des oppositions déjà bien réelles entre le centre et ses périphéries.

Le thème de la centralité reste sans doute le point de crispation principal auquel il faut prêter une attention particulière. La majorité des interviewés évoquent, d'une manière ou d'une autre, le risque de voir émerger, en même temps que des figures vertueuses, de nouvelles catégories repoussoirs : les périurbains et les ruraux qui vivent aux marges et sont soumis à de multiples contraintes. Les interviewés qui se considèrent comme les plus contraints et les plus fragiles du fait de leur distance par rapport à la centralité se perçoivent-ils déjà comme les perdants potentiels ?

Dès lors, le risque est que le scénario construise des frontières entre les zones du territoire mais aussi entre les modes de vie et d'habiter. Certaines zones, principalement les plus lointaines, seraient celles où les comportements sont considérés comme les plus déviants. La concurrence entre les territoires instaurerait alors des formes de stigmatisation où le lointain désigné comme non vertueux serait la catégorie qui doit changer.

Cette représentation est directement en lien avec une question transversale soulevée par le scénario : celle de la centralité. Le rapport à la centralité, comme modèle dominant organisationnel, construit des catégories d'outsiders et laisse peu de marge de manœuvre. Ainsi, les interviewés qui se considèrent comme les plus contraints et les plus fragiles, du fait de leur distance avec la centralité, se perçoivent déjà comme les perdants potentiels.

\footnotetext{
"Je trouve qu'on revient à... j'ai l'impression que dans les années, début du siècle, il y avait une espèce de mixité de population. Les zones de travail étaient proches des lieux de vie, alors qu'à partir des années 60, on a fait du zoning à mort, on a mis des supermarchés, des trucs énormes où on allait consommer, des endroits où on allait travailler, des endroits où on vivait... On n'a pas mélangé les pavillons, les collectifs et tout et j'ai l'impression que toutes ces erreurs-là, j'ai
} 


\section{SHS Web of Conferences}

l'impression qu'on s'en rend compte maintenant. (...). Si les politiques, ils s'entouraient un peu de gens qui pensent... » (Dominique, Centre-Reste noyau urbain)

«Des gens comme nous, on est mobile, on peut marcher... Alors évidemment pour les gens qui ont des problèmes, les personnes âgées ou ceux qui sont en fauteuil, c'est peut-être plus compliqué. (...) on met des barrières, des privilégiés, des non privilégiés, on met des cases, on peut peut-être amener les choses avec plus de souplesse pour les adultes. »(Christelle, Centre-Reste noyau urbain)

Ainsi, que cela soit le surinvestissement de figures vertueuses, celle du centre-ville, ou l'ostracisme attribué aux périphéries, ces perceptions et représentations renvoient à des jugements de valeur, des étiquettes qui ne prennent pas en compte des contraintes de chacun et des différents arbitrages occasionnés en termes de parcours résidentiel, de temps et déplacement professionnels, d'enfants, ... Le risque social identifié est bien celui de voir émerger des groupes sociaux ne participant plus à la constitution de la mixité sociale au sein d'un même territoire. Les périurbains et les ruraux sont perçus et se vivent comme cette catégorie étiquetée non vertueuse, vivant aux marges et soumis à de multiples contraintes.

\section{Conclusion}

Les ménages rencontrés adoptent une attitude plutôt passive face aux changements à venir tout en donnant à voir de multiples stratégies d'adaptation à de nouvelles contraintes et opportunités. On constate que sur de nombreux plans, le mouvement est engagé même s'il se traduit par des expérimentations sans suite (on pense ici par exemple au covoiturage ou à la mutualisation de certains équipements qui ont été largement testés) ou des micro-ajustements qu'il ne faut pas sous-estimer (recours à des modes de déplacement doux, changement dans les modes de consommation, prise de conscience environnementale induisant des comportements plus sobres en énergie...).

\section{Références}

[1] Axes de progrès vers un schéma de cohérence territoriale Facteurs 4. Quels leviers locaux pour une agglomération post-carbone ?, Rapport Final, ATU, Beauvais Consultants, Programme Villes post-carbone, ADEME (janvier 2011)

[2] A. Dujin, B. Maresca, La température du logement ne dépend pas de la sensibilité écologique, CREDOC revue « consommation et modes de vie », $\mathrm{n}^{\circ} 227$ (mars 2008)

[3] Habiter à Tours et travailler à Paris. Étude sur les migrants professionnels, en collaboration avec Beauvais Consultants, PREDIT/DRAST (2007)

[4] Signal Prix et arbitrage de moyen long termes. Comportements des familles face à la hausse du prix du carburant, en collaboration avec Beauvais Consultants, PREDIT (2009) 